\title{
New Approaches to the Surgical Treatment of Lung and Bronchial Tumors in Children
}

\author{
Oleg V. Bilokon ${ }^{1 *}$, Elen V. Shaida ${ }^{1}$, Petro P. Sokur ${ }^{2 \dagger}$, Borys O. Kravchuk ${ }^{2}$ \\ ${ }^{1}$ National Cancer Institute \\ 33/43 M. Lomonosov Str., 03022 \\ Ukraine \\ ${ }^{2}$ P.L. Shupyk National Medical Academy of Postgraduate Education \\ 9 Dorohozhytska Str., 04112 \\ Ukraine
}

Received: February 2, 2021. Revised: June 29, 2021. Accepted: July 23, 2021. Published: July 30, 2021.

\begin{abstract}
Today, it is relevant to search for new, better methods of surgical treatment of tumors and organpreserving and video-assisted surgery is gaining more and more popularity. The aim of the study was to improve the results of surgical treatment of children with primary and secondary (metastatic) lung tumors using high-frequency live tissue welding (HF LTW) in order to increase the treatment effectiveness. Novelty of the study lies in the expanding the knowledge of the new researched method, for enhancement of survivability relatively to chemotherapy and radiation methods, that are studied in the previous works. Advantages of the introduced method are bloodless, fast, low traumatic operations 103 case reports in children with primary and metastatic lung tumors were analyzed, including 34 patients with benign and malignant lung tumors and 69 children with metastatic bronchial and pulmonary lesions undergoing treatment from 2002 to 2018 were examined. Benign lung tumors were diagnosed in 17 patients. Malignant tumors were observed in 17 patients, including 11 lung carcinoid tumors. Metastatic bronchial and pulmonary lesions most often occurred with osteosarcoma (28) and nephroblastoma (17). In 34 children with primary tumors, 37 were undergone surgical interventions. Most often, atypical resection was used $-16(43.3 \%)$, in particular, with carcinoid - in $7(43.8 \%)$ patients and with hamartoma - in $4(25 \%)$ patients. In metastatic lesions, in most cases, the same resection was performed. From 58 resections $25(43 \%)$ were performed for osteosarcoma and $15(26 \%)$ for nephroblastoma. The HF LTW method was used in 29 patients for benign and malignant primary tumors and in $62(73 \%)$ surgical procedures for lung metastases to remove a tumor, vascular coagulation and
\end{abstract}

sealing of the lung tissue. Using HF LTW surgical operations of different directions and volumes can be performed effectively in benign, malignant and metastatic bronchial and pulmonary lesions in children.

Keywords-benign lesions; electric welding; high-frequency live tissue welding; respiratory diseases.

\section{INTRODUCTION}

$\mathrm{T}$ Today, it is relevant to search for new, more effective methods of surgical treatment of tumors and organpreserving and video-assisted surgery is gaining more and more popularity. In particular, the method of precision resection is used, if possible, which is extremely organpreserving, since the targeted tumor removal is performed while preserving the surrounding tissue. Over the past decades, in addition to mechanical methods, modern hightemperature methods have been used in surgical practice to combine the stage of tissue dissection and arrest of bleeding, followed by their direct welding [1-2].

A breakthrough in surgery was the development of electric welding technology (EWT) "Liga Sure", which is based on the passage of a surgical instrument of high strength and low voltage electric current between the branches $[3,4,5]$ which provides denaturation and collagenization of tissues with the vascular thrombus formation. The area of coagulation necrosis is usually $1-1.5 \mathrm{~mm}[3,6]$, not exceeding $2-4 \mathrm{~mm}[7]$. A very important feature of "Liga Sure" EWT is minimal thermal damage of the surrounding tissues, and the presence of a microprocessor in the device ensures that the power supply is terminated when the coagulation effect is achieved [8]. Experimental studies of K. Sugi et al. [9] showed the ability to coagulate vessels with a diameter of $5 \mathrm{~mm}$ using EWT, and the coagulation thrombus withstood a pressure of up to 75 mmHg. For comparison with conventional electrocoagulation, 
we used LigaSure, which significantly reduced the degree of blood loss [10]. Based on the high hemostatic EWT properties, this method could be widely implemented in general [11] and abdominal surgery and gynecology, including laparoscopic surgery [12]. This technology was successfully used when performing splenectomies [13], gastric resections [14], and esophagoectomies [15]. In pulmonary surgery, EWT is used both for open [8] and videothoracoscopic operations [16]. The indications for such interventions were benign tumors and bullous disease [8, 17], and lung cancer and its metastatic lesions $[6,16]$. In Ukraine, the analogue of "Liga Sure" is high-frequency live tissue welding (HF LTW) (using BE Paton's method), which, due to its recent appearance in surgical practice, is being studied and searched for areas of the most appropriate use [18]. Abroad, this technique has been successfully used for minimally invasive precision lung resections and metastasectomies [17, $19,20]$.

For electric welding resections, EK-300 M1 (domestic high-frequency electrocoagulator), developed by E.O. Paton Electric Welding Institute of the National Academy of Sciences of Ukraine in 2007-2008, was effective. The device has the functions of welding, cutting and coagulation, and operates at $66 \mathrm{kHz}$ frequency. The voltage from the electrical network, entering the unit of the device, converts the alternating voltage into direct voltage, then the output voltage is converted by the inverter to high-frequency, transformed and transmitted through the cable to a special welding tool. As a result, the EK-300 M1 performs four functions: automatic electric welding, sealing of blood vessels, HF electric welding of large masses of living tissue, cutting tissue with simultaneous overlapping of blood vessels. The general operational principle of a seamless electric welding machine is the passage of high-strength and low-voltage electric current between the branches of surgical instruments, which provides welding and coagulation effects. Starting at 25 seconds, physiological saline is added to the energy delivery point. This leads to an ordering of the current flow, a decrease in resistance and an increase in the effective input power by 4-5 times. One of the main types of operations carried out with the help of HF LTW is atypical lung resection [21,22] in primary tumors [23].

As for secondary lung tumors, in the vast majority of cases, patients who developed lung metastases were transferred to the category of incurable ones. In recent decades, the literature has discussed the feasibility of combining chemotherapy with the previous surgical removal of isolated intrapulmonary metastases. A certain number of authors [24, 25] believe that surgical treatment of metastases improves the general condition and duration of remission of the existing disease. The survival rate ranges from 17 to $46 \%$ [26, 27]. Currently, the expediency of surgical removal of metastases is recognized by many leading surgeons, and such operations are performed in patients with tumors of various primary localizations. Surgical treatment of intrapulmonary metastases leads to prolongation of the life of patients, especially chemoresistant tumors [28]. Thus, the surgical method remains the leading one in the treatment of bronchial and pulmonary neoplasms; however, different views on the choice of the scope of surgical intervention, the stages and methods of its implementation, indications for surgical treatment of some nosological forms are considered. In particular, the question of the amount of necessary surgical intervention for primary and secondary bronchial and pulmonary tumors in children and the possibility of using the HF LTW method remains unclear.

In course of this study, we analyzed the case reports of children with primary and metastatic lung tumors, then morphological types and frequencies of benign tumors were defined on the basis of analyzed data in the results and discussion section. In the conclusion section, results of the study were introduced, along with recommendations regarding HF LTW method.

\section{MATERIALS AND METHODS}

We retrospectively analyzed the case reports of children with primary and metastatic lung tumors undergoing treatment from 2002 to 2018. The work was carried out in the settings of the clinical site of the Department of Thoracic Surgery and Pulmonology of the P.L. Shupik National Medical Academy of Postgraduate Education of the Ministry of Health of Ukraine and in the Department of Thoracic Surgery for Children of the Kyiv City Clinical Hospital No. 17 and in the site of the Department of Pediatric Oncology of the National Cancer Institute (Kyiv). A total of 103 patients were enrolled in the study, including 34 patients with benign and malignant lung tumors in patients of the Kyiv City Clinical Hospital No. 17 and 69 children with metastatic bronchial and pulmonary lesions among patients of the Pediatric Oncology Department of the National Cancer Institute.

The disease duration (according to clinical signs) ranged from 1 to 5 years. The most common initial symptom was dry cough, mistaken for acute bronchitis. With the growth of the tumor against the background of narrow airways, depending on age, symptoms of obstructive pulmonary emphysema or hypoventilation and atelectasis of the corresponding parts of the lung developed with the subsequent development of organic changes (abscess formation, fibrosis, pneumosclerosis). The main diagnostic method of the studied cohort of patients was plain radiography. Plain radiography in frontal and lateral projections was performed in $103(100 \%)$ patients. The study was carried out on devices TUR -700 (Germany) and EDR - 750 (Hungary). Computed tomography was performed in $70(68 \%)$ patients using a SO-MATOMAR PX computed tomography scanner from Siemens AG (Germany). Magnetic resonance imaging was performed in 8 (7.8\%) patients. The examination was carried out on a Magnetom Symphony tomograph from General Electric (a German company) manufactured in the USA. Frontal, sagittal, and axillary projections were performed at a field strength of $0.5 \mathrm{~T}$ and two modes of operation $-\mathrm{T} 1$ and $\mathrm{T} 2$. Positron 
emission tomography/computed tomography was performed in $48(46.6 \%)$ patients using the Siemens BIOGRAF 40 apparatus. Radiotherapy based on Fluorodeoxyglucose was administered intravenously, the strength was calculated individually, based on the patient's body area. The accumulation time of the radiopharmaceutical was 60-90 minutes. Tracheobronchoscopy was used for diagnostic and therapeutic purposes. A total of 125 different types of bronchoscopy (including pre- and postoperative) were performed in 79 patients $(76.7 \%)$. Preoperative morphological verification was performed in 80 (78.6\%) patients. Postoperative pathomorphological examination was carried out in $103(100 \%)$ patients. Electron microscopic study was performed in $23(22.8 \%)$ patients.

In the case reports studied by us, the treatment of bronchopulmonary tumors is predominantly operative. The choice of access and technique depended on the location of the tumor, its size, and the possibility of malignancy. Treatment of malignant tumors of the bronchi and lungs consisted of the use of surgical methods of treatment, chemotherapy and radiation therapy in various combinations depending on the patient's condition, morphology and stage of the tumor. Surgical treatment was performed to different extent: anatomical or atypical lung resection (wedge-shaped, segmental, etc.), lobectomy, pneumonectomy, precision resections using high-frequency electrosurgical tissue welding. When the tumor process spread to the lymph nodes, extended resections were used. When other organs got involved in the pathological process, resections were combined, in particular, of bronchi and lungs, pericardium. In this case, surgical treatment could be separate (for benign tumors) or combined with chemotherapy, radiation therapy (preoperative, postoperative, palliative). All patients underwent general anesthesia and thoracotomy (lateral and posterior-lateral) access, and two retractors were used for separating wide ribs. When planning surgical interventions, preference was given to organ-preserving methods: atypical resection of the lung, segmentectomy, precision removal of tumors and metastases using HF LTW. After visual and palpatory revision of the lungs, the pathological section of the lung tissue was removed using an organ stapler (VO) or a vascular stapler or using high-frequency electric welding technology according to the selected type of resection, and then underwent immediate histological examination. Depending on the results obtained, the chest was closed or expanded to the required therapeutic volume. After the operation was completed and the chest was sealed, the contents were actively aspirated. The duration of the operations did not exceed 2 hours. No blood loss or complications were observed during or after surgery.

Statistical processing of the obtained data was performed on a personal computer using Excel and Statgraphics-5 software. The arithmetic mean of the indicator (M) and its mean error (m) were calculated. The Disparity between the groups was determined by parametric (Student's t-test) and nonparametric (F-Fisher angular transformation) mathematical statistics methods.

\section{RESULTS AND DISCUSSION}

Benign lesions were observed in $17(50 \%)$ patients out of 34 , malignant - in $17(50 \%)$ children. The evaluation of morphological characteristics specific of solitary pulmonary nodules at conventional imaging studies is essential for appropriate investigation of patients The probability of cancer varies a lot with the size of nodules in the different studied populations. Approximately $80 \%$ of benign nodules are $<2$ $\mathrm{cm}$ in diameter. However, $15 \%$ of the malignant nodules are $<$ $1 \mathrm{~cm}$, and approximately $42 \%,<2 \mathrm{~cm}$. the time spam a malignant nodule takes to double in size is highly variable, generally ranging between 20 and 300 days. Stability over a two-year period implies a doubling time of at least 730 , strongly suggesting benignity The morphological characteristics and frequency of benign tumors were distributed as follows (Table 1).

TABLE I. MORPHOLOGICAL TYPES AND FREQUENCY OF BENIGN TUMORS, $\mathrm{N}=17$

\begin{tabular}{lll}
\hline Tumor type & Abs. & \% \\
\hline hamartoma & 5 & 29.3 \\
\hline adenoma & 4 & 23.5 \\
chondroma & 2 & 11.8 \\
inflammatory pseudotumor & 2 & 11.8 \\
teratoma & 1 & 5.9 \\
fibroma & 1 & 5.9 \\
fibropapilloma & 1 & 5.9 \\
hemangiolymphangioma & 1 & 5.9 \\
Total & $\mathbf{1 7}$ & $\mathbf{1 0 0}$ \\
\hline
\end{tabular}

Table 1 showed that most common conditions in children were: hamartoma (5 (29.3\%)) and adenoma (4 (23.5\%)), which is typical for primary lung tumors in pediatric patients. The morphological characteristics and frequency of primary malignant tumors were as follows (Table 2).

\section{TABLE II. MORPHOLOGICAL TYPES AND FREQUENCY OF} MALIGNANT TUMORS, $\mathrm{N}=17$

\begin{tabular}{lll}
\hline Tumor type & Abs. & \% \\
\hline carcinoid & 11 & $64.6 \%$ \\
neurosarcoma & 1 & $5.9 \%$ \\
teratoneuroblastoma & 1 & $5.9 \%$ \\
neuroendocrine tumor & 1 & $5.9 \%$ \\
non-Hodgkin lymphoma & 1 & $5.9 \%$ \\
adenocarcinoma & 1 & $5.9 \%$ \\
malignant mesenchymoma & 1 & $5.9 \%$ \\
Total & $\mathbf{1 7}$ & $\mathbf{1 0 0 \%}$ \\
\hline
\end{tabular}

Table 2 shows that the absolute majority of primary malignant lung tumors in children were carcinoid (11 $(64.6 \%))$. These data are in full agreement with the literature [29]. 
The age range of patients with benign and primary malignant tumors ranged from 3 to 18 years. The largest proportion (50\%) includes teenagers of 13-18 years old (17 patients). The second ranking place was taken by children of 8-12 years old $-11(32 \%)$, and the third place by children of preschool age (3-7 years old) $-6(18 \%)$. Thus, there is a direct correlation between the age of a child and the incidence of primary benign and malignant tumors. Regarding the gender distribution of patients, the proportion of girls (19 patients $(55.9 \%))$ slightly exceeded that among boys (15 (44.1\%)), but this disparity was not significant $(p>0.05)$. As for the localization of the primary lesion of the lungs, the majority of patients had right lung lesions (19 children (56\%)), which was 1.8 times higher than the number of patients with left lung lesion (10 children $(32 \%, \mathrm{p}<0.05))$, and 4.7 times with bilateral lesion ( 5 children $(12 \% ; \mathrm{p}<0.001)$ ).

As we noted, primary lung tumors are rare. Most often, the lungs and bronchi are affected as a result of metastasis of neoplasms of other organs and tissues. Our study thoroughly analyzed 69 children with metastatic lesions of the bronchi and lungs among the patients of the Pediatric Oncology Department of the National Cancer Institute. The morphological structure and frequency of secondary (metastatic) malignant tumors are presented below (Table 3).

TABLE III. MORPHOLOGICAL TYPES AND FREQUENCY OF METASTATIC TUMORS, $\mathrm{N}=69$

\begin{tabular}{lll}
\hline Tumor type & Abs. & $\mathbf{\%}$ \\
\hline osteosarcoma & 28 & $41 \%$ \\
nephroblastoma & 17 & $25 \%$ \\
embryonal rhabdomyosarcoma & 4 & $6 \%$ \\
hepatoblastoma & 4 & $6 \%$ \\
malignant teratoblastoma & 3 & $5 \%$ \\
neuroblastoma & 3 & $5 \%$ \\
ganglioneuroblastoma & 2 & $1.5 \%$ \\
peripheral primitive tumor & 2 & $1.5 \%$ \\
myofibroblastic tumor & 1 & $1 \%$ \\
biphasic synovial sarcoma & 1 & $1 \%$ \\
immature malignant teratoma & 1 & $1 \%$ \\
epidermoid carcinoma & 1 & $1 \%$ \\
embryonal carcinoma & 1 & $1 \%$ \\
renal cell carcinoma & 1 & $1 \%$ \\
Total & $\mathbf{6 9}$ & $\mathbf{1 0 0 \%}$ \\
\hline
\end{tabular}

According to our data (Table 3), metastatic lesions of the bronchi and lungs most often occurred in osteosarcoma (28 $(41 \%))$ and nephroblastoma (17 (25\%)) and much less often in embryonal rhabdomyosarcoma and hepatoblastoma (4 (6\%)).

The distribution of patients with metastatic tumors of the lungs and bronchi in relation to the localization of the primary lesion is shown in Table 4.
TABLE IV. DISTRIBUTION OF PATIENTS WITH METASTATIC TUMORS OF THE LUNGS AND BRONCHI IN RELATION TO THE LOCALIZATION OF THE PRIMARY LESION, $\mathrm{N}=69$

\begin{tabular}{lll}
\hline Localization & Abs. & \% \\
\hline Femur & 18 & $26.1 \%$ \\
Kidneys & 18 & $26.1 \%$ \\
Humerus & 6 & $8.7 \%$ \\
Liver & 4 & $5.7 \%$ \\
Fibula & 4 & $5.7 \%$ \\
Thoracic cavity & 3 & $4.3 \%$ \\
Mediastinum & 2 & $2.8 \%$ \\
Adrenal glands & 2 & $2.8 \%$ \\
Forearm soft tissues & 2 & $2.8 \%$ \\
Ovaries & 1 & $1.5 \%$ \\
Testicles & 1 & $1.5 \%$ \\
Small pelvis & 1 & $1.5 \%$ \\
Salivary glands & 1 & $1.5 \%$ \\
Retroperitoneal space & 1 & $1.5 \%$ \\
Thigh soft tissues & 1 & $1.5 \%$ \\
Sacrum soft tissue & 1 & $1.5 \%$ \\
Groin soft tissues & 1 & $1.5 \%$ \\
Parotid region & 1 & $1.5 \%$ \\
Tibia & 1 & $1.5 \%$ \\
\hline Total & $\mathbf{6 9}$ & $\mathbf{1 0 0 \%}$ \\
\hline
\end{tabular}

As follows from Table 4, in the majority (26.1\%) of patients, the primary localization is the femur and kidneys. In addition, the first four in frequency included lesions of the humerus $(8.7 \%)$, fibula and liver $(5.7 \%$ each). The age of patients with metastatic lung tumors ranged from 1 to 18 years. The largest proportion of patients $(53.6 \%)$ was at the age of 10-13 years. The second ranking place is occupied by patients aged 5-9 years (30.4\%), the lowest was the proportion of patients aged 1-4 years (16\%), which significantly differed from other age intervals. In general, there is a direct correlation between age and disease incidence. With regard to gender differences in patients, the proportion of boys was 39 children $(57 \%)$, and girls $-30(43 \% ; p<0.001)$. Analysis of the localization of lung lesions with secondary neoplasms showed that most often (in 41 patients, $60 \%$ ) tumors occurred in both lungs. Right lung lesion was 2.6 times less frequent (in 16 patients, $22.8 \%, \mathrm{p}<0.001$ ), and left lung lesion was 3.5 times less frequent (in 12 patients, $17 \%, \mathrm{p}<0.001$ ) than in bilateral lesions.

In the case reports analyzed in patients with benign and malignant lung tumors, the following types of resections were performed (Table 5).

\section{TABLE V. TYPES OF SURGICAL INTERVENTIONS IN 34 CHILDREN} WITH PRIMARY LUNG TUMORS

\begin{tabular}{lll}
\hline Type and extent of surgical intervention & Abs. & \% \\
\hline Atypical resection & 16 & $43.3 \%$ \\
Circular resection & 6 & $16.2 \%$ \\
Pulmonectomy & 5 & $13.5 \%$ \\
Lobectomy & 3 & $8.1 \%$
\end{tabular}




\begin{tabular}{lll} 
Fenestrated resection & 3 & $8.1 \%$ \\
Marginal resection & 1 & $2.7 \%$ \\
Pleural bilobectomy & 1 & $2.7 \%$ \\
Bronchoscopic tumor removal & 1 & $2.7 \%$ \\
Precision resection & 1 & $2.7 \%$ \\
Total & 37 & $100 \%$ \\
\hline
\end{tabular}

Based on data, atypical resection is the most common type of resection in lung operations [30]. All types of atypical resections involve the use of a stapler. The lung parenchyma is stitched with a stapler within healthy tissues, and the resection of the pathologically altered part of the lung after the stitching is performed with a scalpel. The stapler sutures the vascular structures and bronchus only along the edge of the area of pathologically altered tissues and, thus, preserves unchanged (healthy) parts of the lung segment, which would have to be removed during a typical resection. The most common is a mechanical metal (tantalum) double-row suture, which provides a quick and adequate seal and hemostasis when separating the tissue of the lung parenchyma. But hemostasis and hermeticity with this method of suturing are not always sufficiently provided. Therefore, recently, resections using a high-frequency electrosurgical welding machine have been widely used $[31,32]$.

As can be seen from Table 6, 37 surgical interventions were performed in 34 children with primary tumors. Most often, atypical resection was used (16 (43.3\%)), in particular, for carcinoid (in 7 (43.8\%) patients) and for hamartoma (in 4 (25\%) patients). Circular resection was used for bronchial tumors $(6(16.2 \%))$, most often for carcinoid (in three (50\%) patients), lobectomy was used only for carcinoid (3 (8.1\%)), and pulmonectomy (5 (13.5\%)) and pleural bilobectomy (1 $(2.7 \%)$ ) for large tumors and advanced forms. Most of the children received only surgical treatment. Only one child with bilateral teratoneuroblastoma in the inferior lobe of the right lung and metastases in the inferior lobe of the left lung had chemotherapy. In children with metastases to the lungs from other tumors, a different distribution of surgical interventions types was observed (Figire 1).

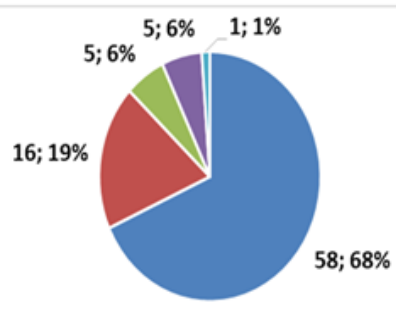

$\begin{array}{ll}\text { | Atypical resection } & \text { | Precision resection } \\ \text { | Lobectomy } & \text { " Segmental resection }\end{array}$

FIGURE 1. TYPES OF RESECTIONS IN 69 CHILDREN WITH METASTASIS OF OTHER TUMORS TO THE LUNGS (TOTAL NUMBER OF OPERATIONS - 85)

Thus, in most cases, an atypical lung resection was performed. Of 58 resections $(68 \%$ of the total number of operations), 25 (43\%) were performed for osteosarcoma, and
$15(26 \%)$ for nephroblastoma. Precision tumor resection was performed 16 times (19\%), mainly in osteosarcoma (6 $(37.5 \%))$ and nephroblastoma (4 (25\%)). Marginal resection (5 (6\%)) was performed in $40 \%$ of patients with teratoblastoma. Pulmonectomy was not performed, but lobectomy was performed in $(5(6 \%))$ patients with nephroblastoma $(40 \%)$.

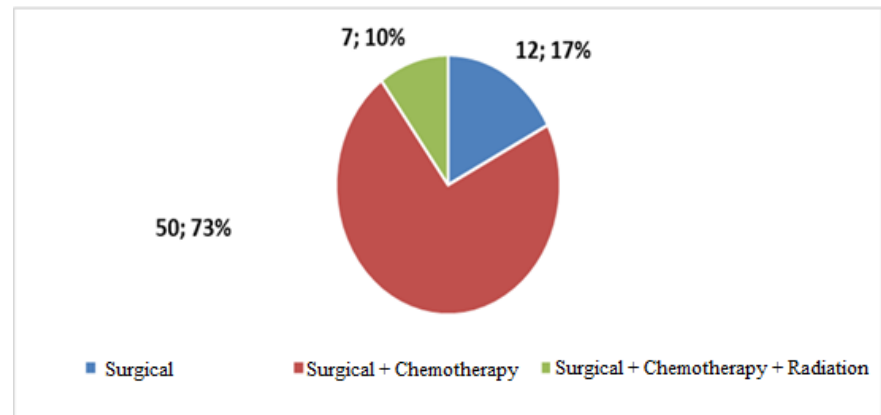

FIGURE 2. TYPES OF TREATMENT FOR CHILDREN WITH METASTATIC TUMORS OF THE LUNGS AND BRONCHI, $\mathrm{N}=69$

As can be seen from Figure 2, the majority of children (50; $73 \%$ ) underwent surgical removal of the tumor, followed by courses of corresponding chemotherapy. In 12 children (17\%), surgical removal of metastases was sufficient. Moreover, in 2 children (4\%), surgical intervention was carried out according to the palliative program, and in $48(96 \%)$ according to the radical program. The rest of the patients underwent a complex of surgical treatment, chemotherapy and radiation therapy. It should be noted that of 69 children hospitalized with lung metastases in 2000-2018, 31 (45\%) were diagnosed with stage 4 of the disease, which indicates a low level of tumor diagnostics in childhood.

\section{CONCLUSION}

In the case reports analyzed, the HF LTW method was used in 29 patients (in $78 \%$ of surgical interventions) with benign and malignant primary neoplasms and in 62 (73\%) surgical interventions for lung metastases to remove the tumor, coagulate blood vessels and seal the lung tissue, which indicates the widespread use and effectiveness of this method.

When using the HF LTW method, thoracotomy was performed according to one of the generally recognized techniques. The extent and limits of the lung area to be removed were determined according to the usual sequence of actions for atypical resection. In the designated places, a bipolar clamp was inserted and applied, connected to the EK 300 M1 biological welding complex in the "welding" mode, within healthy tissues. At the same time, the process was based on the general principles of preserving resection and general rules for electrocoagulation to prevent damage to surrounding tissues. Welding of the lung parenchyma was performed until an adequate suture of the lung was fully formed, followed by the resection of the pathologically altered portion of the lung with a cutting tool. Additionally, we used a set of clamps and tweezers developed at the B.E. Paton 
Research Institute of Electric Welding of the National Academy of Sciences of Ukraine, adapted for seamless electrosurgery.

Bronchoplastic operations were performed with an unchanged lung, as well as with reversible changes in the lung distal to the tumor (aseptic atelectasis, deforming bronchitis, obstructive emphysema, retention bronchiectasis) also using the HF LTW method. A feature of fenestrated and wedgeshaped resections of the bronchus with a tumor is minimal trauma to the peribronchial neurovascular branches outside the operating area. In case of arising defects of the bronchial wall, when suturing without deformation of the lumen was impossible, a circular resection of the bronchial segment was performed, followed by anastomosis. For circular resection of the bronchus with lobectomy, the bronchial tubes were mobilized more neutral and distal to the tumor, and then bronchoanastomosis was formed. The tightness of the suture line in all cases was controlled by a water sample. Lung resection or pulmonectomy was performed in cases of late diagnosis of the disease, complicated by abscess formation of the lung or fibroatelectasis.

Using the HF LTW method, surgical operations of various directions and extent for benign, malignant and metastatic lesions of lungs and bronchi in children can be performed. The HF LTW method used for surgical bronchial and pulmonary interventions reduces the volume of intraoperative blood loss, decreases the duration of the operation and the number of complications, and improves the functional capacity of the lung tissue. These provide its promising and advantageous use in thoracic surgery and in primary and secondary lung and bronchial tumors in children.

\section{ACKNOWLEDGMENT}

We are grateful to Petro P. Sokur for help in writing this article. We offer the whole family sincere condolences in connection with the irreparable loss.

\section{References}

[1] A. V. Makarov, V. G. Getman, and A. V. Linchevsky. "Possibilities of using the technique of "welding of living tissue" in thoracic surgery", In: Collection of Abstracts of Reports (pp. 23-24). Kyiv: Institute of Electric Welding E.O. Paton NAS of Ukraine, 2012.

[2] R. K. Nadirov, K. S. Nadirov, G. Zh. Bimbetova, and Zh. K. Nadirova, "Synthesis and cytotoxic activity of new flavopiridol analogs," Chem. Nat. Comp., vol. 52, pp. 499-500, 2016.

[3] K. G. Zhestkov, and Yu. S. Esakov. "Seamless lung resection with the LigaSure device: possibilities and prospects," Surgery, vol. 4, pp. 30-35, 2012.

[4] O. Kovasc, Z. Szanto, G. Kraznai, and G. Hezz, "Comparing bipolar electrothermal device and endostapler in endoscopic lung wedge resection," Interactive CardioVasc. Thorac. Surg., vol. 9, pp. 11-14, 2009.
[5] I. I. Spyroglou, E. A. Chatzimichail, E. N. Paraskakis, and A. G. Rigas, "A comparison of different ridge parameters in an asthma persistence prediction model," Int. J. Biol. Biomed. Eng., vol. 10, pp. 1-9, 2016.

[6] D. E. Alexandrov, and S. A. Nersisyan, "Identification of contact angle and heterogenity detection in tactile images,” Int. J. Biol. Biomed. Eng., vol. 12, pp. 186-191, 2018.

[7] L. Bertolaccini, A. Viti, A. Cavallo, and A. Tezzi, "Results of the trial: a prospective randomized study on effectiveness of "LigaSure" in lung resections," Eur. J. Cardio-Thorac. Surg., vol 45, pp. 693-698, 2014.

[8] M. J. Schuchert, G. Abbas, and B. L. Pettiford, "Preliminary results of anatomic lung resection using energy-based tissue and vessel coagulative fusion technology," J. Thorac. Cardiovasc. Surg., vol. 140, no. 5, pp. 1168-1173, 2010.

[9] K. Sugi, Y. Kaneda, Y. Satoh, and T. Murakami, "Effects of bipolar vessel sealing system in pulmonary rezections," Japan. J. Thorac. Cardiovasc. Surg., vol. 56, pp. 551-554, 2003.

[10] S. Say, N. P. Dugos, S. A. Roces, and J. M. Mondragon, "Sonication-assisted perfusion decellularization of whole porcine kidney,” Int. J. Biol. Biomed. Eng., vol. 13, pp. 78-81, 2019.

[11] A. V. Sikorsky, M. O. Welcome, and V. A. Pereverzev, "Lipid metabolism and functions of pituitary-thyroid axis in children with arterial hypotension," Int. J. Biol. Biomed. Eng., vol. 12, pp. 124-130, 2018.

[12] I. Mazic, A. Bjelopera, and L. Strazicic, "Comparison of the SVM classification results between original and DWT denoised respiratory signals considering to the transient's noise," Int. J. Biol. Biomed. Eng., vol. 12, pp. 143-150, 2018.

[13]F. Romano, R. Caprotti, and C. Franciosi, "Laparoscopic splenectomy using "LigaSure" preliminary experience," Surg. Endoscop., vol. 16, pp. 1608-1611, 2002.

[14] W. J. Lee, T. C. Chen, and I. R. Lai, "Randomized clinical trial of "LigaSure" versus conventional surgery for extended gastric cancer resection," British J. Surg., vol. 90, pp. 1493-1496, 2003.

[15]E. Yekeler, H. Ulutas, C. Becerik, and K. Peher, "The use of "LigaSure" in esophagectomy," Interact. Cardio-Vasc. Thorac. Surg., vol. 11, pp. 10-14, 2010.

[16] A. Watanabe, M. Migajima, N. Kawaharada, and T. Higami, "Two separate thoracoscopic segmentectomies with vessel sealing system," Europ. J. Cardio-Thorac. Surg., vol. 41, pp. e62-e64, 2012.

[17]N. Shigemura, A. Akashi, and T. Wakagiri, "A new tissue-sealing technique using the "LigaSure" system for nonanatonical pulmonary resection: preliminary results of suturelles and stapless thoracoscopic surgery," Annals Thorac. Surg., vol. 77, pp. 1415-1418, 2004.

[18]B. E. Paton, "Electric welding of soft tissues in surgery," Automat. Weld., vol. 9, pp.7-11, 2004.

[19] V. Koryahin, O. Blavt, N. Bakhmat, M. Guska, T. Ludovyk, M. Prozar, A. Bodnar, S. Kravets, and E. Bezgrebelnaya, "Differentiated correction of attention abilities of students with chronic diseases during physical 
education,” J. Phys. Educ. Sport, vol 19, no 2, pp. 293298, 2019.

[20] A. Yumashev, I. Semenycheva, B. Rakhadilov, and A. Tsymbal, "Development of biocompatible coatings for dental implants based on transition metal nitrides," J. Glob. Pharma Technol., vol. 11, no. 5, pp. 22-28, 2019.

[21]B. E. Paton, and O. N. Ivanova, "Tissue-preserving highfrequency electric welding surgery". Kyiv: Naukova Dumka, 2009.

[22] A. V. Makarov, V. G. Hetman, and O. V. Linchevsky, "Application of the method of electrical connection of living tissues in thoracic surgery". In: Materials of the 5th Ukrainian-Polish Scientific-Practical Conference Dedicated 100th Anniversary of Thoracosurgery (pp. 250-252). Wroclaw: Wroclaw Medical University, 2006.

[23] V. Koryahin, O. Blavt, N. Bakhmat, M. Guska, T. Ludovyk, M. Prozar, A. Bodnar, S. Kravets, and E. Bezgrebelnaya, "Differentiated correction of attention abilities of students with chronic diseases during physical education,” J. Phys. Educ. Sport, vol. 18, no. 4, pp. 22782283, 2018.

[24] B. K. Poddubny, "Results of surgical treatment of metastatic lung tumors," Thorac. Surg., vol. 4, pp. 52-57, 1969.

[25] W. Alakwaa, M. Nassef, and A. Badr, "Lung cancer detection and classification with 3D convolutional neural network (3D-CNN),” Int. J. Biol. Biomed. Eng., vol. 11, pp. 66-73, 2017.

[26] U. Pastorino, M. Valente, and M. Gasparini, "Median Sternotomy and multiple lung resections for metastatic sarcomas," Europ. J. Cardio-Thorac. Surg., vol. 4, no. 9, pp. 477-481, 1990.

[27] U. Pastorino, "History of the surgical management of pulmonary metastases and development of the international registry," Seminars Thorac. Cardiovasc. Surg., vol. 14, pp. 18-28, 2002.

[28] A. V. Linchevsky, A. V. Makarov, B. A. Kravchuk, P. P. Sokur, and V. Getman, "The use of precision bipolar instruments for videothoracoscopy in adults and children". In: VIII International Scientific and Practical Conference - Welding and Heat Treatment of Living Tissues. Theory. Practice. Prospects (pp. 158-170). Kyiv: E.O. Paton Electric Welding Institute, 2013.

[29] N. S. Opanasenko, B. N. Konik, V. I. Klimenko, V. A. Kononenko, A. V. Tereshkovich, M. I. Kalenichenko, V. B. Bychkovsky, R. S. Demus, O. K. Obremska, L. I. Levanda, and A. E. Kshanovsky, "Carcinoid tumor of lung: literature review and own observation," Ukrainian Pulmonol. J., vol. 4, pp. 48-53, 2013.

[30] P. C. A. Yim, E. A. Rendina, and S. R. Hazelrigg, “A new technological approach to nonanatomical pulmonary resection: saline enhanced thermal sealing," Annals Thorac. Surg., vol. 74, pp. 1671-1676, 2002.

[31]D. I. Sternbergand, and J. R. Sonett. "Surgical therapy of lung metastases," Seminars Oncol., vol. 34, pp. 186-196, 2007.

[32] O. I. Smiyan, Y. A. Man'ko, A. M. Loboda, S. V. Popov, I. Y. Vysots'kyy, V. O. Petrashenko, I. M. Martsovenko, K. O. Smiian, V. A. Plakhuta, V. M. Serhiyenko, O. L.
Ovsyanko, T. O. Aleksakhina, and A.-R. Bara, "Influence of magnesium on the state of the cardiovascular system in children with chronic tonsilitis," Wiadom. Lekar., vol. 73, no. 5, pp. 904-908, 2020.

[33] I. S. Mavromatakis, S. G. Liliopoulos, and G. S. Stavrakakis, "Optimized intermittent pharmaceutical treatment of cancer using non-linear optimal control techniques," WSEAS Trans. Biol. Biomed., vol. 17, no. 9, pp. 67-75, 2020.

[34]Z. Sultana, Md. A. R. Khan, and N. Jahan, "Early breast cancer detection utilizing artificial neural network," WSEAS Trans. Biol. Biomed., vol. 18, no. 4, pp. 32-42, 2021.

[35]H. A. M. Vyshnavi, and P. K. K. Namboori, "Identification of lapatinib derivatives and analogs to control metastatic breast cancer-specific to South Asian population - a pharmacogenomic approach," WSEAS Trans. Biol. Biomed., vol. 18, no. 6, pp. 51-62, 2021.

\section{Author Contributions:}

Oleg V. Bilokon: Conceptualization, Investigation, Formal analysis and Writing - original draft.

Elen V. Shaida: Investigation, Formal analysis and Writing - original draft and Writing - review \& editing. Petro P. Sokur: Investigation, Formal analysis and Writing - original draft and Writing - review \& editing. Borys O. Kravchuk: Investigation, Formal analysis and Writing - original draft and Writing - review \& editing.

\section{Creative Commons Attribution License 4.0 (Attribution 4.0 International , CC BY 4.0)}

This article is published under the terms of the Creative Commons Attribution License 4.0

https://creativecommons.org/licenses/by/4.0/deed.en US 